\title{
Gold Nanoparticles Enhance EGFR Inhibition and Irradiation Effects in Head and Neck Squamous Carcinoma Cells
}

\author{
Masahiko Kashin, ${ }^{1}$ Yasumasa Kakei ${ }^{1},{ }^{1}$ Shun Teraoka, ${ }^{1}$ Takumi Hasegawa, ${ }^{1}$ \\ Akinobu Yamaguchi, ${ }^{2}$ Takao Fukuoka, ${ }^{3}$ Ryohei Sasaki, ${ }^{4}$ and Masaya Akashi ${ }^{1}$ \\ ${ }^{1}$ Department of Oral and Maxillofacial Surgery, Kobe University Graduate School of Medicine, Kobe 650-0017, Japan \\ ${ }^{2}$ Laboratiory of Advanced Science and Technology for Industry, University of Hyogo, Kamigori 678-1205, Japan \\ ${ }^{3}$ Graduate School of Engineering, Kyoto University, Kyoto 615-8540, Japan \\ ${ }^{4}$ Division of Radiation Oncology, Kobe University Graduate School of Medicine, Kobe 650-0017, Japan
}

Correspondence should be addressed to Yasumasa Kakei; ykakei@med.kobe-u.ac.jp

Received 21 July 2020; Revised 7 September 2020; Accepted 19 October 2020; Published 7 November 2020

Academic Editor: Jane Hanrahan

Copyright @ 2020 Masahiko Kashin et al. This is an open access article distributed under the Creative Commons Attribution License, which permits unrestricted use, distribution, and reproduction in any medium, provided the original work is properly cited.

Cetuximab, an epidermal growth factor receptor inhibitor (EI), is currently the only targeted molecular therapy used in combination with radiotherapy for head and neck squamous cell carcinoma (HNSCC). Gold nanoparticles (AuNPs) are expected to enhance radiotherapy effects in cancers. To investigate whether AuNPs combined with AG1478, an EI, enhanced irradiation effects on HNSCC cells, we first examined AG1478 adsorption on AuNP surfaces, using surface-enhanced Raman scattering, which indicated an adsorption equilibrium of AG1478 to AuNPs. We then used transmission electron microscopy to find internalization rates of AuNP alone and AuNP+AG1478; we found that intracellular uptake of AuNP alone and AuNP +AG1478 did not significantly differ. We compared cell numbers, proliferation, apoptosis, and migration between control cells and those treated with or without $60 \mathrm{~nm}$ AuNP $(1.0 \mathrm{nM})$, AG1478 $(0.5 \mu \mathrm{M})$, and irradiation (4Gy). We found that AuNP +AG1478 inhibited proliferation more than AG1478 alone; the combination of irradiation+AuNP+AG1478 significantly reduced total cell numbers compared with the combination of irradiation+AuNP; AuNP+AG1478 increased apoptotic reaction to irradiation; the combinations of AuNP+AG1478 and irradiation+AuNP induced more apoptosis than AG1478+irradiation. Whereas AuNP+AG1478 enhanced cytotoxicity in human HNSCC cells by inhibiting proliferation, irradiation+AuNP enhanced cytotoxicity by inducing apoptosis.

\section{Introduction}

Over $90 \%$ of all head and neck cancers are squamous cell carcinomas, and $90 \%-100 \%$ of head and neck squamous cell carcinomas (HNSCCs) overexpress epidermal growth factor receptor (EGFR) [1]. Overexpression of EGFR is correlated with decreased overall and disease-free survival and increased resistance to radiation therapy (RT) and locoregional relapse [2-4].

EGFR inhibitors (EIs) have been tested against HNSCC in several randomized clinical trials. In 2006, cetuximab, a chimeric antibody $(\mathrm{Ab})$ that targeted EGFR, was included in HNSCC regimens after a phase III trial and showed a survival benefit for cetuximab+RT compared with RT alone [5]. Since 2008 , the regimen of cetuximab+platinum +5 -fluorouracil has been the first-line standard of care for HNSCC, based on a randomized trial [6]. Although cetuximab enhanced the effects of RT and chemotherapy in these studies, results were disappointing, as only $10 \%-30 \%$ of patients responded to monotherapy. This implies some intrinsic resistance [7]. A novel strategy to improve response by $\mathrm{EGFR}^{+}$HNSCC is therefore needed.

Recently, nanoparticle- (NP-) based approaches have been investigated for improving cancer response to both RT and chemotherapy [8]. Noble metal NPs have been introduced as novel drug delivery systems for anticancer therapies [9-12]. Gold nanoparticles (AuNPs) have attracted particular interest as they are easy to synthesize in various sizes and shapes and to functionalize with biomolecules that, together with their particular optical properties, may have 
both therapeutic and diagnostic applications in oncology [13]. Additionally, because of gold's high atomic number and well-established biosafety, AuNPs may be suitable as radiosensitizing agents [14-17].

AuNPs easily combine with many biological ligands, such as single antibodies or peptides, which can be used as probes to selectively recognize cancer $[18,19]$. Thus, AuNPs could potentially be functionalized with anti-EGFRAb to treat EGFR-overexpressing tumors such as HNSCCs. AntiEGFR monoclonal $\mathrm{Ab}$ (mAb; EGFRmAb) conjugated to AuNPs (EGFRmAb-AuNP) can reportedly induce apoptosis in squamous laryngeal and breast cancer cell lines $[20,21]$. However, as the effects of radiation+EGFRmAb-AuNP in HNSCC cells are unclear, we used cell counting, proliferation, and wound healing assays and apoptosis counts to evaluate this combination on HNSCC cells; surface-enhanced Raman scattering (SERS) to study the adsorption of EI +AuNP; and transmission electron microscope (TEM) analysis to assess the uptake ratio of EI+AuNP.

\section{Materials and Methods}

2.1. Cell Culture. We used the human HNSCC cell line HSC3 (tongue carcinoma; Japanese Collection of Research Bioresources Cell Bank, Tokyo, Japan). The cells were cultured in Dulbecco's modified Eagle's medium (DMEM; Wako Pure Chemical Industries, Ltd., Osaka, Japan) supplemented with $10 \%(\mathrm{v} / \mathrm{v})$ fetal bovine serum (Biowest, Nuaillé, France) and $1 \%$ penicillin/streptomycin (Sigma-Aldrich; Merck KGaA, Darmstadt, Germany) at $37^{\circ} \mathrm{C}$ in a humidified atmosphere of $5 \%(\mathrm{v} / \mathrm{v}) \mathrm{CO}_{2}$. Cells were incubated in DMEM for $48 \mathrm{~h}$ prior to treatment. We used cells that were 2-10 passages from their primary culture.

2.2. Reagents and Antibodies. Citrate-stabilized AuNPs were purchased from Cytodiagnostics, Inc. (Burlington, ON, Canada). Standard AuNPs (60 nm) were used (lot number: 2458052_60). Rat anti-E-cadherin mAb (ECCD2) was purchased from Takara Bio, Inc. (Otsu, Japan), and the tyrosine kinase inhibitor, AG1478, was obtained from Merck KGaA (Darmstadt, Germany). Secondary antibodies conjugated with Cy3 donkey anti-mouse IgG (H+L; AP192C) were purchased from Invitrogen (Thermo Fisher Scientific, Inc., Waltham, MA, USA). Cleaved caspase-3 Ab was purchased from Cell Signaling Technology (Danvers, USA).

2.3. Detection of Adsorption of AG1478 on AuNPs. We used SERS on the tyrosine kinase inhibitor AG1478 in solution with AuNP colloids to determine whether AG1478 is adsorbed on the surfaces of AuNPs. In SERS, the intensity of Raman scattering is greatly amplified when a molecule is adsorbed on a rough surface, such as a precious metal, compared with bulk molecules, by a factor of $10^{4}-10^{6}[22,23]$. In 1997, Nie et al. made it possible to use SERS spectroscopy to measure a single protein molecule with AuNPs, which was a dramatic step forward in this technique [24]. SERS techniques require a noble metal nanostructure. Gold or silver nanoparticles are usually used because they are capable of excitation of the essentially strong localized surface plasmon resonance; AuNPs, in particular, are also chemically stable, generally inert, and are easy to use [25].

For the AuNPs, we used a $60 \mathrm{~nm}$ gold colloid, reduced with citric acid. AG1478 (5 mg) was dissolved in $1 \mathrm{~mL}$ ethanol for a solution of $\sim 15.8 \mathrm{mM}$ and diluted sequentially with ethanol to make AG1478 solutions of $1 \mathrm{mM}, 100,10,3,2,1 \mu \mathrm{M}$, and $500 \mathrm{nM}$ concentrations in $1 \mathrm{~mL}$ Eppendorf tubes. In an approximately $100 \mu \mathrm{L}$ capacity aluminum pan, we mixed AG1478 ethanol solution $(3 \mu \mathrm{L}), 1 \mathrm{M} \mathrm{NaCl}(3 \mu \mathrm{L})$, and AuNP $(54 \mu \mathrm{L})$ by pipetting $[26,27]$ and coaggregated the mixture to generate self-assembled anisotropic structures with SERS activity. Coaggregation allows the absorption spectra of the liquid to converge to the same shape, so that the electric field enhancement by localized plasmon resonance will be almost the same and quantitative measurement of intensity of Raman scattering can be expected [28, 29]. Additionally, the measurement can be stable for about 10 min until the agglomeration settles down. Concentration of this solution becomes $5 \%$. It was examined with a simple Raman spectroscope, RAM100S (Lambda Vision), equipped with a laser of $785 \mathrm{~nm}(100 \mathrm{~mW})$. The solution was placed in an aluminum pan and exposed, 10 times, to radiation for $0.1 \mathrm{~s}$ or $1 \mathrm{~s}$. All experiments were performed at room temperature.

2.4. TEM Images of Cellular AuNP Uptake. To determine whether binding AuNPs to AG1478 affected AuNP uptake, lysosomal uptake of AuNP was measured by TEM. HSC-3 cells were cultured with $60 \mathrm{~nm}$ AuNPs (50 nM), both alone and as a mixture of $60 \mathrm{~nm}$ AuNP (50 nM) and AG1478 $(0.5 \mu \mathrm{M})$. The samples were fixed with $2 \%$ paraformaldehyde and $2 \%$ glutaral aldehyde (GA) in $0.1 \mathrm{M}$ phosphate buffer (PB) $\mathrm{pH} 7.4$ at incubation temperature and put into a refrigerator for $30 \mathrm{~min}$ to lower the temperature to $4^{\circ} \mathrm{C}$. Thereafter, they were fixed with $2 \% \mathrm{GA}$ in $0.1 \mathrm{M} \mathrm{PB}$ at $4^{\circ} \mathrm{C}$ overnight. After fixation, the samples were washed three times with $0.1 \mathrm{M} \mathrm{PB}$ for $30 \mathrm{~min}$ each and postfixed with $2 \%$ osmium tetroxide $\left(\mathrm{OsO}_{4}\right)$ in $0.1 \mathrm{M} \mathrm{PB}$ at $4^{\circ} \mathrm{C}$ for $1 \mathrm{~h}$. The samples were dehydrated in an alcohol gradient, then transferred to a resin (Quetol-812; Nisshin EM Co., Tokyo, Japan), and polymerized at $60^{\circ} \mathrm{C}$ for $48 \mathrm{~h}$. The polymerized resins were sectioned at $80 \mathrm{~nm}$ with a diamond knife, using an ultramicrotome (Ultracut UCT; Leica, Vienna, Austria), mounted on copper grids, stained with $2 \%$ uranyl acetate for $15 \mathrm{~min}$, washed with distilled water, and secondary stained with lead stain solution (Sigma-Aldrich Co., Tokyo, Japan) for $3 \mathrm{~min}$. The grids were visualized by a TEM (JEM-1400Plus; JEOL Ltd., Tokyo, Japan) at an acceleration voltage of $100 \mathrm{kV}$. Digital images $(3296 \times 2472$ pixels) were taken with a CCD camera (EM14830RUBY2; JEOL Ltd.).

2.5. Gold Nanoparticles, AG1478, and Irradiation. Cells were grown in $2 \mathrm{~mL}$ DMEM on glass slips in $35 \times 10 \mathrm{~mm}$ polystyrene tissue culture dishes at a density of $1 \times 10^{5}$ cells $/ \mathrm{mL}$. Cells were incubated with AuNPs, diluted with $1.0 \mathrm{nM}$ phosphate-buffered saline (PBS) and AG1478 at 0 or $0.5 \mu \mathrm{M}$. After $24 \mathrm{~h}$ at $37^{\circ} \mathrm{C}$, samples were irradiated by a MBR-1505R2 X-ray generator (Hitachi, Tokyo, Japan) at $150 \mathrm{kV}, 4 \mathrm{~mA}$, using a $1 \mathrm{~mm}$ aluminum filter. Cells were exposed to a fixed dose of $4 \mathrm{~Gy}$, as reported previously [30]. 
Cells were incubated at $37^{\circ} \mathrm{C}$ for $48 \mathrm{~h}$, after which experiments were conducted.

2.6. Cell Counting by Immunofluorescence Staining and Microscopy. Cells were grown with DMEM on glass slips in $35 \times 10 \mathrm{~mm}$ polystyrene tissue culture dishes at a density of $1 \times 10^{5}$ cells $/ \mathrm{mL}$ for $24 \mathrm{~h}$. We added AG1478 (0 [control] or $0.5 \mu \mathrm{M})$ and AuNPs (0 [control] or $1 \mathrm{nM}$ ) to each well. After $24 \mathrm{~h}$ incubation, cells were irradiated. After $48 \mathrm{~h}$ irradiation, we fixed with $1 \%$ formaldehyde in PBS for $10 \mathrm{~min}$, treated with $0.2 \%$ Triton X-100 in PBS for $5 \mathrm{~min}$ and washed with PBS, then blocked with $1 \%$ bovine serum albumin (BSA; Sigma-Aldrich; Merck KGaA) in PBS for 30 min and incubated with primary antibodies (rat anti-E-cadherin $\mathrm{mAb}$ ) for $24 \mathrm{~h}$ at $4^{\circ} \mathrm{C}$. All antibodies were diluted with $1 \% \mathrm{BSA}$ in PBS, then rinsed three times with PBS and stained with $4^{\prime}, 6-$ diamidino-2-phenylindole (DAPI; Thermo Fisher Scientific, Inc., Waltham, MA, USA) and incubated with corresponding secondary antibodies $\left(1: 500\right.$; Cy $\left.3^{\circledR}\right)$ for $30 \mathrm{~min}$. After rinsing with PBS, specimens were embedded in FluorSave ${ }^{\mathrm{TM}}$ (Merck $\mathrm{KGaA}$ ). They were then viewed with a BZ-X 700 fluorescence microscope (Keyence Corporation, Osaka, Japan). Cell numbers were calculated by counting $\mathrm{DAPI}^{+}$nuclei; to count cell junctions, numbers of cell-cell borders in which E-cadherin was expressed were counted in 10 randomly selected microscopic images (magnification $\times 20$ ). Results were expressed as averaged data of three independent experiments.

2.7. Apoptosis. To detect apoptotic cells, cells were grown with DMEM on glass slips in $35 \times 10 \mathrm{~mm}$ polystyrene tissue culture dishes at a density of $1 \times 10^{5}$ cells $/ \mathrm{mL}$ for $24 \mathrm{~h}$. We added AG1478 (0 [control] or $0.5 \mu \mathrm{M}$ ) and AuNPs (0 [control] or $1 \mathrm{nM}$ ) to each well. After a 24 -hour incubation, cells were irradiated. Forty-eight hours after the irradiation, we fixed with $1 \%$ formaldehyde in PBS for 10 min, treated with $0.2 \%$ Triton $\mathrm{X}-100$ in PBS for 5 min and washed with PBS, then blocked with $1 \%$ bovine serum albumin (BSA; Sigma-Aldrich; Merck $\mathrm{KGaA}$ ) in PBS for $30 \mathrm{~min}$ and incubated with primary antibodies (rat anti-E-cadherin $\mathrm{mAb}$ ) for $24 \mathrm{~h}$ at $4^{\circ} \mathrm{C}$. All antibodies were diluted with $1 \%$ BSA in PBS. The cells were then rinsed three times with PBS and stained with caspase-3 (Cell Signaling Technology, Danvers, USA) and 4',6-diamidino2-phenylindole (DAPI; Thermo Fisher Scientific, Inc., Waltham, MA, USA) and incubated with corresponding secondary antibodies $\left(1: 500\right.$; $\left.\mathrm{Cy}^{\circledR}{ }^{\circledR}\right)$ for $30 \mathrm{~min}$. After rinsing with PBS, specimens were embedded in FluorSave ${ }^{\mathrm{TM}}$ (Merck $\mathrm{KGaA}$ ) and observed under a fluorescence microscope. Apoptotic cells were calculated as a percentage of total cells.

2.8. Cell Survival. Growth inhibitory effects of AuNP, AG1478, and irradiation were evaluated. Numbers of viable cells were determined by using a Cell Counting Kit- 8 (CCK-8; Dojindo Molecular Technologies, Inc., Kumamoto, Japan) according to the manufacturer's instructions. We suspended $5 \times 10^{3}$ cells in complete medium, in a 96-well plate $(100 \mu \mathrm{L} /$ well $)$ at $37^{\circ} \mathrm{C}$. After $24 \mathrm{~h}$, we added AG1478 and/or AuNPs at the above-described concentrations to each well. After a 24-hour incubation, cells were irradiated. We added CCK-8 solution $(10 \mu \mathrm{L})$ to cells $48 \mathrm{~h}$ after irradiation. Plates were then incubated for $1-4 \mathrm{~h}$, after which absorbance was measured at $450 \mathrm{~nm}$ using a microplate reader.

2.9. Wound Healing Assay. Cells $\left(3 \times 10^{5}\right.$ cells $\left./ \mathrm{mL}\right)$ were plated in duplicate in 6-well plates, grown to $70 \%-80 \%$ confluence, and incubated with high-glucose DMEM that contained AG1478 (0 [control] or $0.5 \mu \mathrm{M})$ and AuNPs (0 [control] or $1 \mathrm{nM})$. After $24 \mathrm{~h}$, cells were exposed to irradiation (0 [control] or $4 \mathrm{~Gy})$. After $48 \mathrm{~h}$, cell monolayers were scraped with sterile $200 \mu \mathrm{L}$ disposable plastic pipette tips and washed with PBS. Wound healing was checked with microscopy at $0,4,8,12,16,20$, and $24 \mathrm{~h}$ at $37^{\circ} \mathrm{C}$ after wounding. Images were taken with a BZ-X700 fluorescence microscope (Keyence Corporation; $4 \mathrm{x}$ magnification), and area was calculated by an analysis software BZ-H4M (Keyence).

2.10. Statistical Analysis. Each of our experiments was performed in triplicate with results expressed as mean \pm standard deviation. Statistical analyses were performed using the R software (R Development Core Team, 2011). One-way analysis of variance and Tukey-Kramer multiple comparison tests were used on cell counting, cell survival, and apoptosis data. $P<0.05$ was considered significant.

\section{Results}

3.1. Comparison of Solid Raman Spectra. We initially used SERS measurements to see whether AG1478 is adsorbed on the surfaces of AuNPs. First, we checked the Raman spectrum of AG1478 in its solid state and in its liquid phase with citric acid-reduced AuNPs. Figure 1(a) shows the comparison of Raman spectra of citrate-capped AuNPs without AG1478 (blue dashed line), $500 \mathrm{nM}$ AG1478 in ethanol solution with AuNPs (red solid line), and spontaneous Raman of AG1478 solid (green solid line). As shown in Figure 1(a), when the citratecapped AuNPs alone were aggregated, peaks of citric acid adsorbed on the surface appeared at $1580 \mathrm{~cm}^{-1}$ (unadsorbed citrate ion $\mathrm{C}=\mathrm{O}$ stretching asymmetrically in $\mathrm{COO}^{-}$), $1535 \mathrm{~cm}^{-1}$ (adsorbed citrate ion Au-COO${ }^{-}$stretching), and $1295 \mathrm{~cm}^{-1}$ (adsorbed citrate ion [blue dashed line]), whereas a clearly different spectrum (red line) was observed in the $500 \mathrm{nM}$ AG1478 solution, in which the main peaks appeared at $1590,1480,1370,1260,1190$, and $970 \mathrm{~cm}^{-1}$. However, in measuring the Raman spectra of AG1478 solids with another Raman microspectrometer (green line), the main peaks appeared at 1590,1498, and 1470;1390, 1330, and 1290; 1160 ; and $980 \mathrm{~cm}^{-1}$. By comparing with these spectra, the main peak positions of AG1478 in ethanol (red solid line) are expressed near those of solids and are different from the Raman spectrum of the citrate-treated AuNPs. As the spectrum shape is slightly changed from that of the solid because it is dissolved in ethanol, the spectrum observed for the $500 \mathrm{nM}$ solution (red solid line) in the presence of AuNPs was considered to be the SERS spectrum derived from AG1478.

3.2. Sample Mixing Order. AG1478 is a water-insoluble molecule that may adsorb to the aluminum of its container. Therefore, we tried adding AuNPs to an aluminum pan first, followed by the AG1478 ethanol solution, and $1 \mathrm{M} \mathrm{NaCl}$ in that order. At $500 \mathrm{nM}$, the spectra were almost the same 


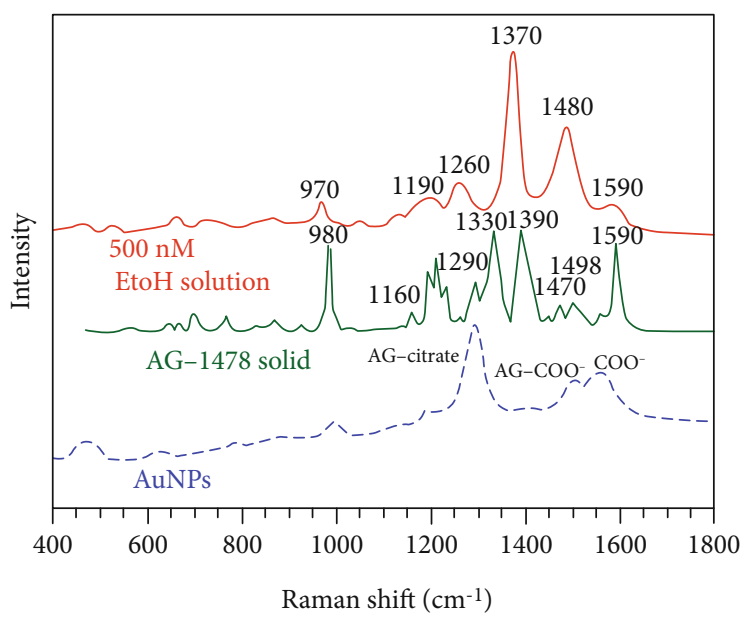

(a)

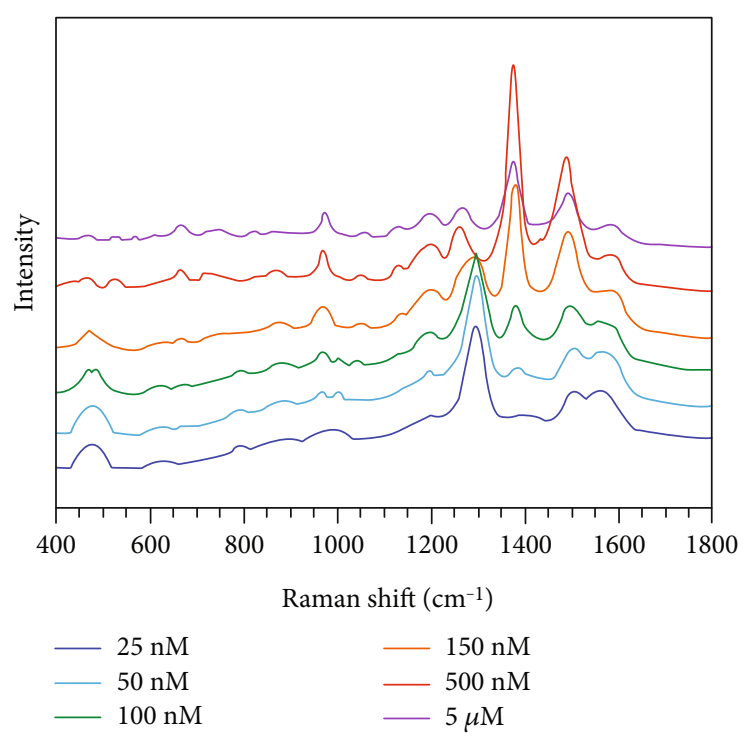

(c)

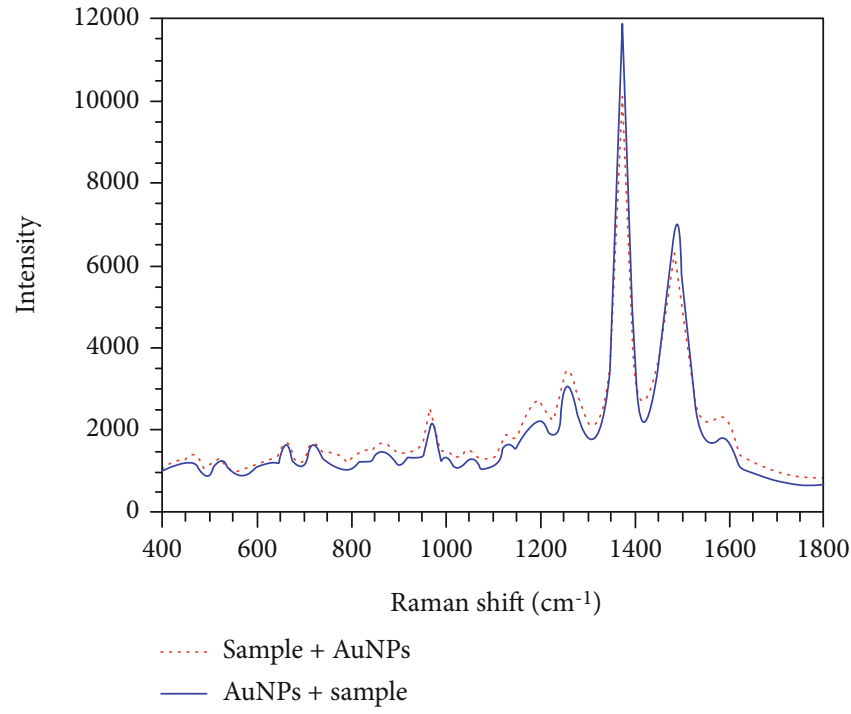

(b)

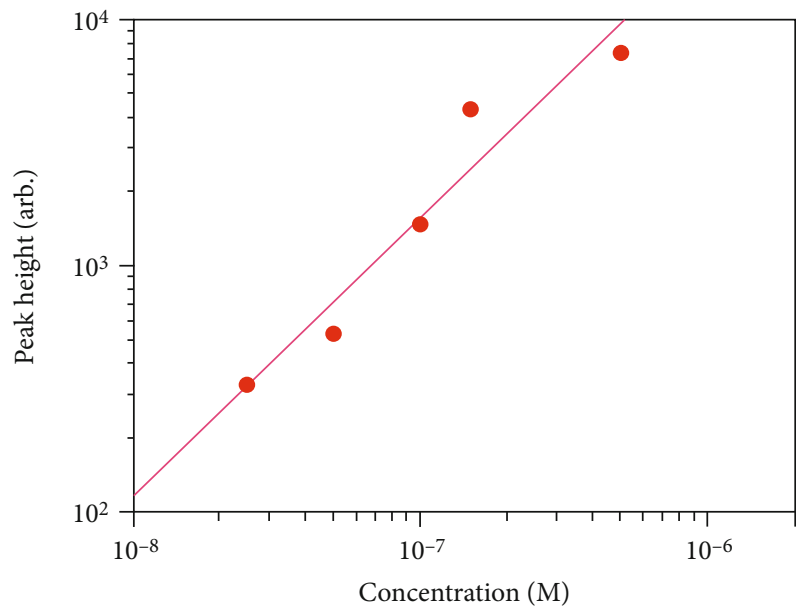

(d)

Figure 1: (a) Enhanced Raman spectrum of AG-1478 $500 \mathrm{nM}$ EtOH/solution, normal Raman spectrum of solid, and citrate-capped AuNPs. (b) Enhanced Raman spectrum when the order of mixing AG-1478 $500 \mathrm{nM} \mathrm{EtOH/solution} \mathrm{and} \mathrm{AuNPs} \mathrm{was} \mathrm{changed} \mathrm{from} \mathrm{front} \mathrm{to} \mathrm{back.} \mathrm{(c)}$ Enhanced Raman spectra of AG1478 concentrations at $5 \mu \mathrm{M}, 500,150,100,50$, and $25 \mathrm{nM}$ in the measured solution. (d) [Log]-[log] plot of the SERS peak height at $1370 \mathrm{~cm}^{-1}$ by concentration yielded nearly sigmoid-like calibrations in the range of 500, 150, 100, and 50 nM AG1478.

whether AuNPs were added first or later (Figure 1(b)). However, adding AG1478 before adding $\mathrm{NaCl}$ caused aggregation, which allowed for the formation of self-assemblies with the same localized plasmon resonance. This aggregation induced by AG1478 can affect the formation of highly sensitive SERS-active self-assemblies induced by the coaggregation method. Therefore, we continued to add AuNPs later in the following experiments.

3.3. SERS and Calibration Curves for Ethanol Solution. Spectra of AG1478 concentrations at $5 \mu \mathrm{M}, 500,150,100,50$, and $25 \mathrm{nM}$ in the measured solution are shown in Figure 1(c). From $5 \mu \mathrm{M}$ to $50 \mathrm{nM}$, characteristic peaks appeared at 1480 ,
1370,1260 , and $970 \mathrm{~cm}^{-1}$, depending on the concentration. At concentrations below $500 \mathrm{nM}$, the peak at $1295 \mathrm{~cm}^{-1}$ from the citric acid adsorbed on the AuNPs was stronger. At $25 \mathrm{nM}$, almost no peak from AG1478 could be observed. A [log]-[log] plot of the SERS peak height at $1370 \mathrm{~cm}^{-1}$ by concentration yielded nearly sigmoid-like calibrations in the range of 500, 150, 100, and $50 \mathrm{nM}$ (Figure 1(d)). The appearance of enhanced citric acid peaks at low concentrations, and this sigmoidal behavior suggests an adsorption equilibrium between AG1478 and AuNPs.

3.4. Intracellular Uptake of AuNPs by Adding AG1478. TEM showed dispersed gold particles in endocytic vesicles, but 


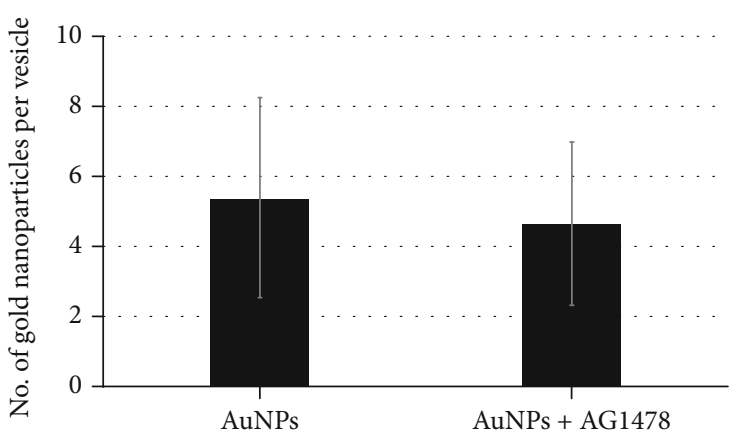

(a)
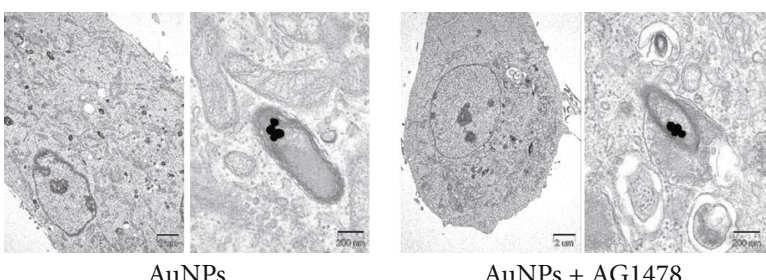

AuNPs + AG1478

(b)

Figure 2: (a) Adding AG1478 did not increase numbers of AuNPs in cluster-forming vesicles. The actual amount of AuNP uptake did not significantly differ between the AuNP-alone and AG1478+AuNP groups. (b) Representative TEM images. AuNP clusters consisted of several AuNPs.

none were detected in the nucleus. Numbers of AuNPs in vesicles that formed clusters did not significantly differ between the AuNP-alone and AG1478+AuNP groups (Figure 2(a)). Figure 2(b) shows representative TEM images. AuNP clusters consisted of several AuNPs.

3.5. Cell Counting Assay. We assessed the effects of three concentrations of $60 \mathrm{~nm}$ AuNPs without X-rays in HSC-3 cells. Cell numbers did not significantly differ between the control cells and those treated with 1.0, 10, or $50 \mathrm{nM}$ AuNPs (data not shown). Representative immunohistochemistries of eight groups, with or without $1 \mathrm{nM}$ AuNP, $0.5 \mu \mathrm{M}$ AG1478, and 4 Gy irradiation, are shown in Figure 3(a).

Compared with the control group and the AuNP-alone group, the other six groups all showed significant decreases in cell numbers (Figure 3(b)). Moreover, the $0.5 \mu \mathrm{M}$ AG1478+1 nM AuNP+4 Gy irradiation group had significantly lower cell counts than the $1 \mathrm{nM}$ AuNP+4 Gy irradiation group $(P<0.001$; Figure 3(b)).

3.6. Proliferation Assay. We used proliferation and apoptosis assays to establish the underlying causes of the decreased cell numbers in the irradiation+AuNP+AG1478 group. Compared with AuNP alone, the AuNP+AG1478 groups, both with irradiation $(P=0.02)$ and without irradiation $(P=0.03$ ), were significantly reduced on cell growth (Figure 4(a)).

3.7. Measurement of Apoptosis. The combination of AuNP +irradiation induced apoptosis, as shown by the significantly greater percentage of caspase $-3^{+}$cells, compared with the AuNP-alone, AG1478-alone, irradiation-alone, and irradiation+AG1478 groups $(P<0.001$; Figure $4(\mathrm{~b}))$. Moreover, the AuNP+AG1478+radiation had a significantly greater apoptotic percentage compared with AuNP+irradiation $(P<0.001$; Figure 4(b)).

3.8. In Vitro Wound Healing Assay. In Figure 5(a), the area was calculated from the area enclosed in green, which was automatically analyzed by Keyence's software BZ-H4M, according to the area of the central part of the scratch, excluding the missing part of the cell.

Biological responses to EGFR signaling are pleiotropic and can result in tumor-promoting processes, including enhanced cell motility [31]. Adding AuNP and/or irradiation to AG1478 reduced cell motility compared with AG1478 only (Figure 5(b)).

\section{Discussion}

This study revealed that AuNP+AG1478+irradiation led to the greatest reduction of HSC-3 cell numbers among the eight possible combinations of these treatments. Whereas reduction of cell numbers by AuNPs adsorbed on AG1478 was caused by suppression of cell proliferation, reduction of cell numbers by radiation+AuNP was caused by apoptosis.

To our knowledge, this is the first study of radiosensitization by AuNP adsorption on an EI against human HNSCC cells. However, Dahai et al. showed radiosensitization using silver nanoparticles combined with EI in nasopharyngeal carcinoma [32].

AuNPs show promise for clinical applications and may potentially be used for RT and photodynamic therapy because of their biostability, ease of surface modification, and radiation sensitization. In the present study, we confirmed the adsorption of AG1478 to the surface of AuNPs by Raman spectroscopy, but similar results were reported in previous reports with other EIs such as gefitinib and erlotinib $[33,34]$. The chemical formula of AG1478 is similar to those of gefitinib and erlotinib, which suggests that the quinazoline ring portion is bound to the gold surface $[33,34]$.

The combination of AG1478+AuNP reduced cell proliferation compared with AG1478 only (Figure 4(a)); and AuNP+AG1478 reduced cell migratory activity compared with AG1478 only (Figure 5). As described in previous reports, the decreased migratory activity was probably caused by adhesion of drugs such as EGFR or HER2 inhibitors onto the surfaces of the AuNPs, which enhanced their anticancer effect compared with EGFR or HER2 inhibitor alone [35-37].

Uptake of AuNPs into HSC-3 cells by AG1478 addition was analyzed by TEM, but the AuNPs with and without AG1478 did not significantly differ (Figure 2(b)). However, Kubota et al. reported that HER2 inhibitor conjugated to AuNPs was efficiently internalized into cells, and this HER2dependent internalization may affect the cytotoxic mechanisms of trastuzumab+AuNP [37]. As measuring gold particles 

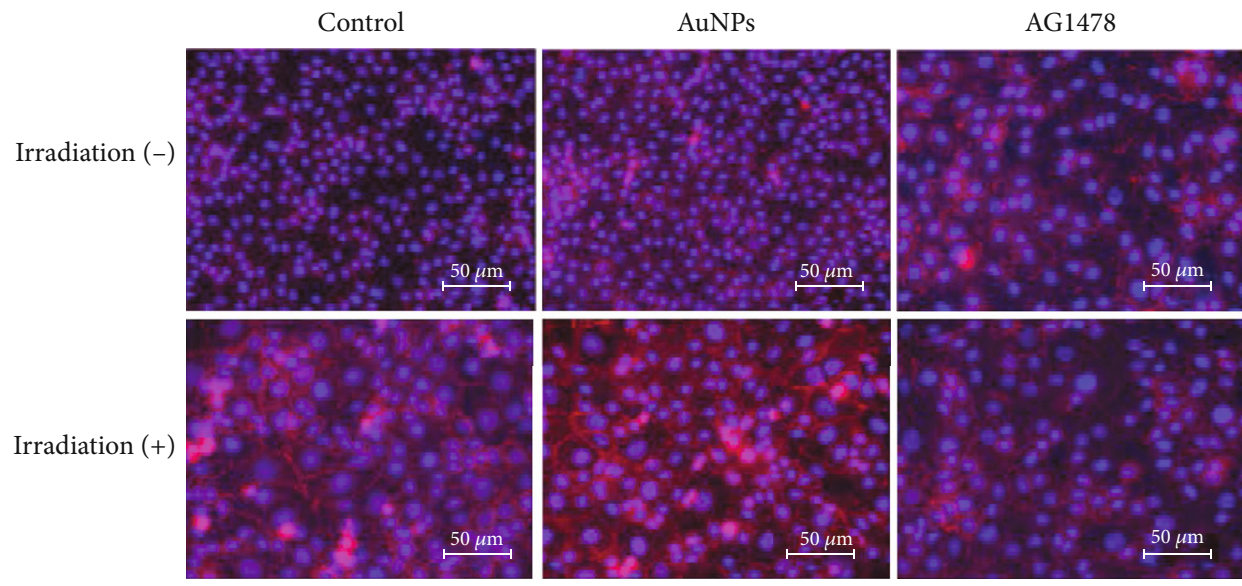

AuNPs + AG1478

(a)

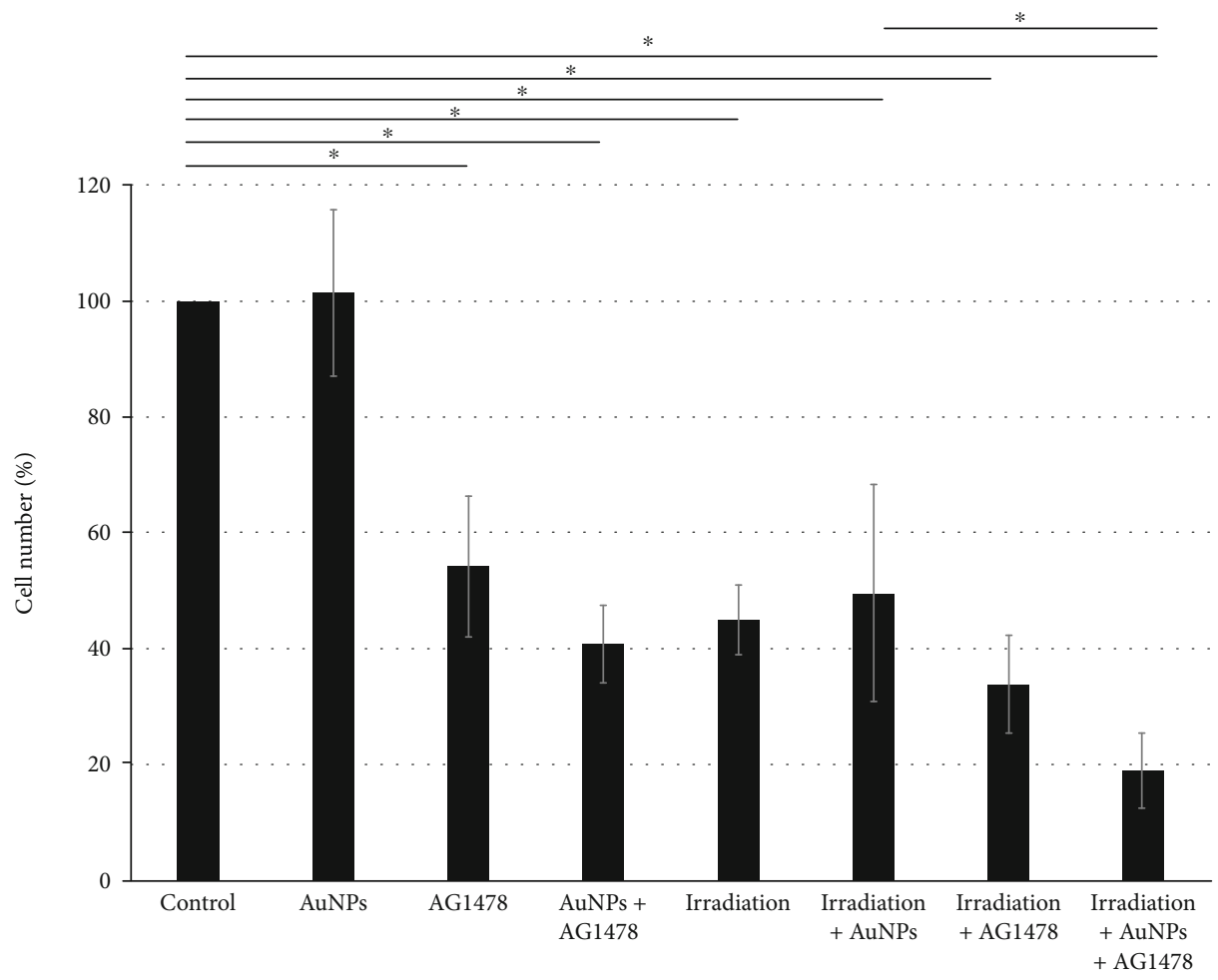

(b)

Figure 3: (a) Effects of the combination of $1 \mathrm{nM}$ AuNP+0.5 $\mu \mathrm{M}$ AG1478 on HNSCC cell proliferation, with or without 4 Gy irradiation. For counting, cells and their nuclei were visualized using DAPI and E-cadherin. (b) Compared with the control group and the AuNP-alone group, the other six groups all showed significantly decreased cell numbers. In addition, the $0.5 \mu \mathrm{M}$ AG1478+1 nM AuNP+4 Gy irradiation group had significantly lower cell counts than the $1 \mathrm{nM}$ AuNP+4 Gy irradiation group.

smaller than $60 \mathrm{~nm}$ is difficult by the coaggregation method, AuNPs of $60 \mathrm{~nm}$ were used in this study. However, AuNPs of $40-50 \mathrm{~nm}$ are reportedly the most effective, and Kubota et al. [37] used $50 \mathrm{~nm}$ AuNPs. A previous study by Jiang et al. [38] reported that, although the AuNPs were found inside the cytoplasm in the trastuzumab-40 nm AuNP-treated cells, the AuNPs were found only on the cell surface in the trastuzumab-70 nm AuNP-treated cells. This result indicates that in cells treated with antibody+larger AuNPs (i.e., larger than $60 \mathrm{~nm}$ ), little or no AuNPs are present in the cytoplasm. In our study, AuNP internalization into cells was confirmed with
TEM, but why adding AG1478 had no effect on AuNP internalization should be addressed in the future research.

Although adding AG1478 to AuNPs did not increase apoptosis, the rate of apoptosis was increased by irradiation (Figure 4(b)). Binding AG1478 to AuNP surfaces did not apparently inhibit the radiosensitizing effect of AuNPs on apoptosis. Although increased apoptosis after radiation and AuNPs has been widely reported, including our own report [30], we found no other reports of irradiation and AuNPs bound to EIs in HNSCC. Melancon et al. reported that near-infrared laser irradiation of gold nanoshells conjugated 


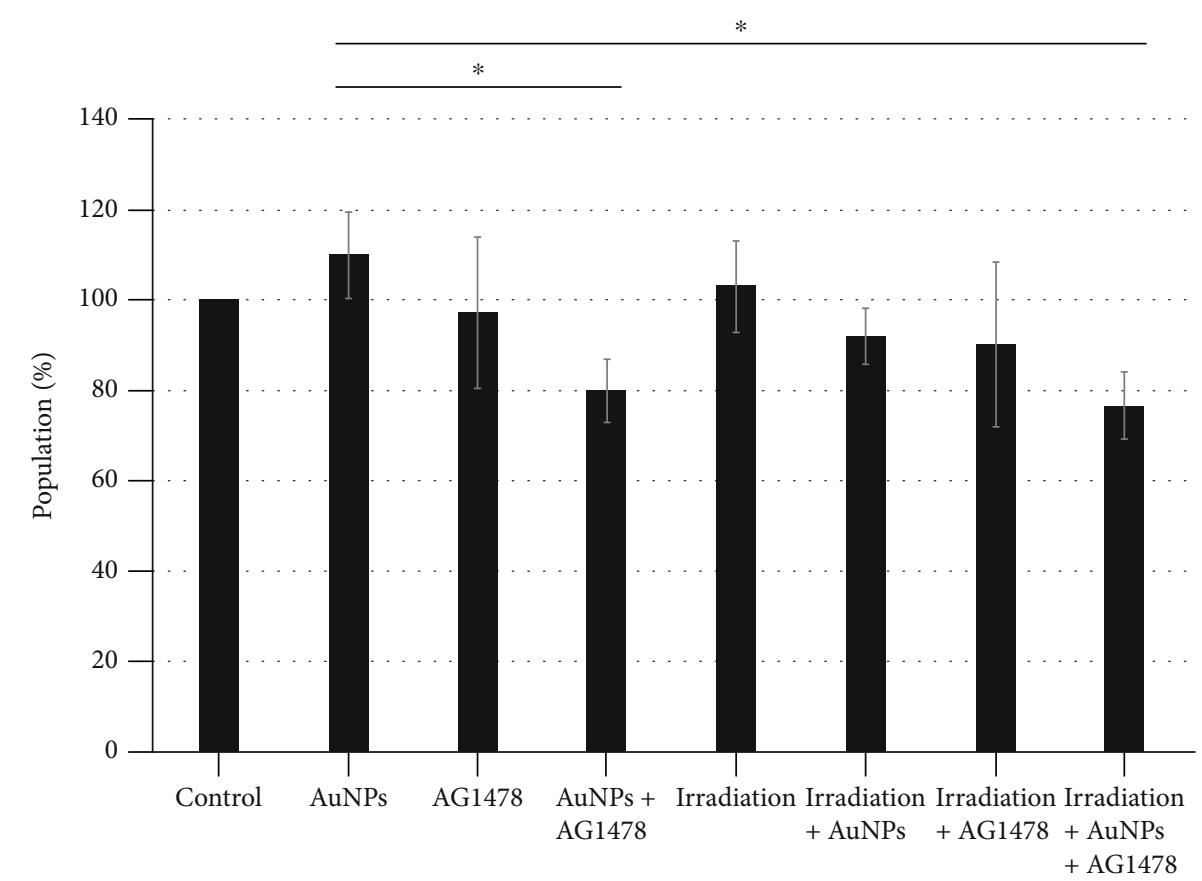

(a)

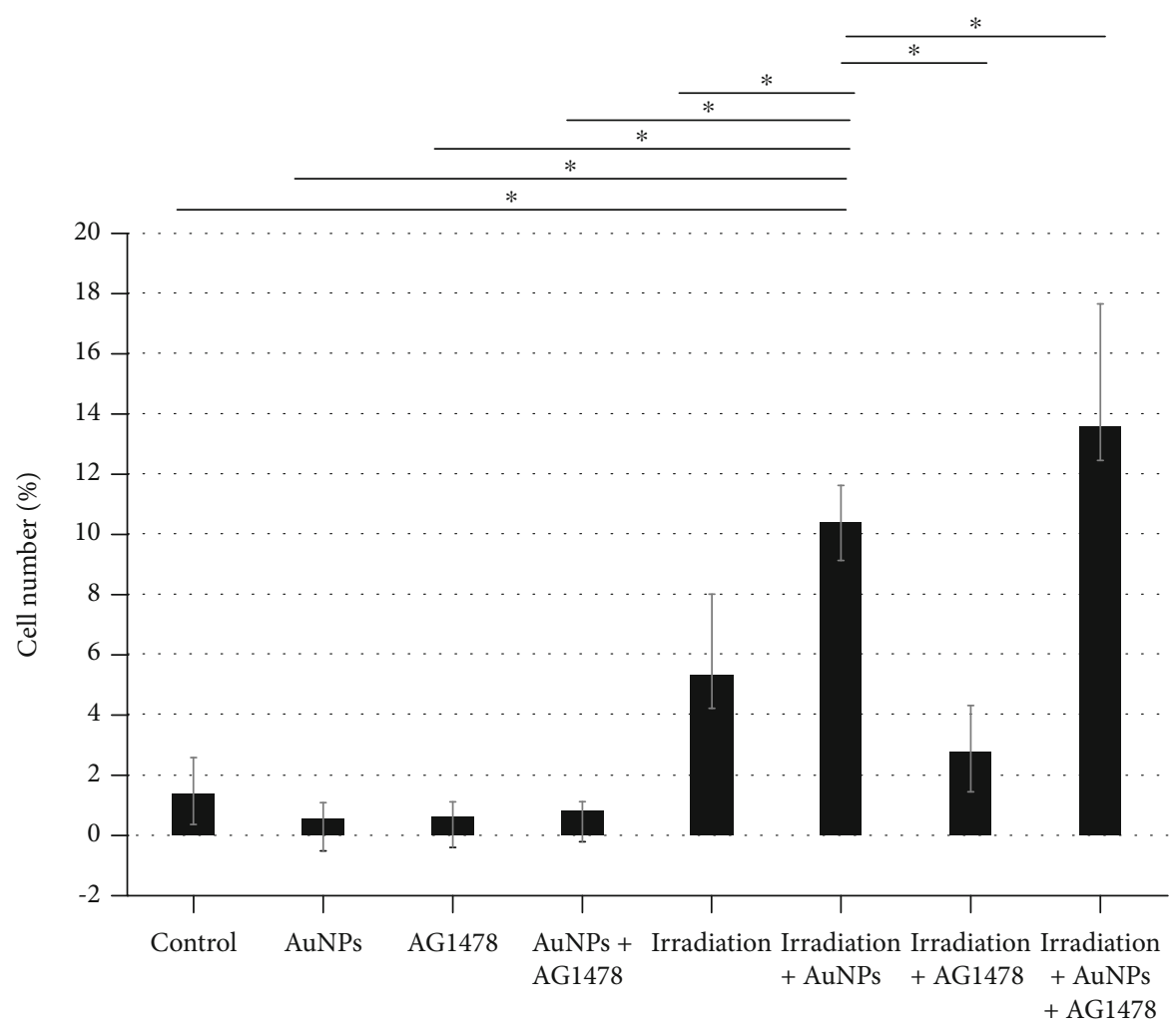

(b)

FIGURE 4: (a) Adding AuNPs and AG1478 and/or irradiation significantly inhibited cell proliferation. Cell Counting Kit-8 assay shows the combination of $1 \mathrm{nM}$ AuNP+0.5 $\mu \mathrm{M}$ AG1478 inhibited HNSCC cell proliferation, both with and without 4 Gy irradiation. Y-axis values indicate percentages, with the control as $100 \%$. Data represent three independent experiments and are presented as means \pm standard deviation. AuNPs: gold nanoparticles. (b) Adding AuNPs and/or AG1478 significantly enhanced the apoptotic effect of irradiation. The effects of combining $1 \mathrm{nM}$ AuNPs+0.5 $\mu \mathrm{M}$ AG1478 on apoptosis in HNSCC cells, with or without 4 Gy irradiation, significantly increased the percentage of capase $-3^{+}$(apoptotic) cells compared with $4 \mathrm{~Gy}$ irradiation alone. $Y$-axis values are expressed as percentages, with the control as $100 \%$. The data represent three independent experiments and are presented as means \pm standard deviation. 


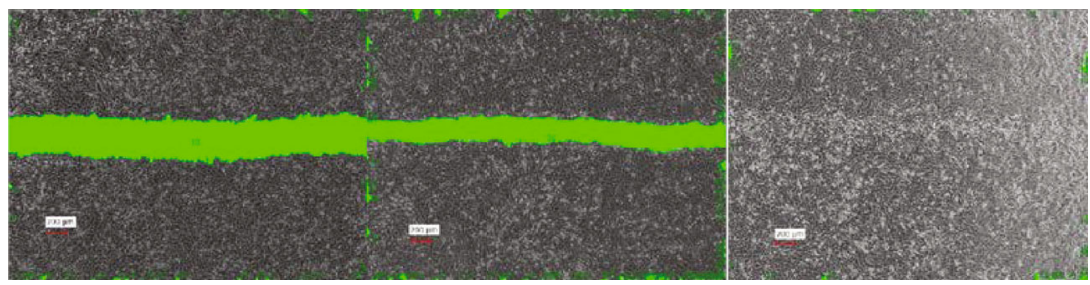

(a)

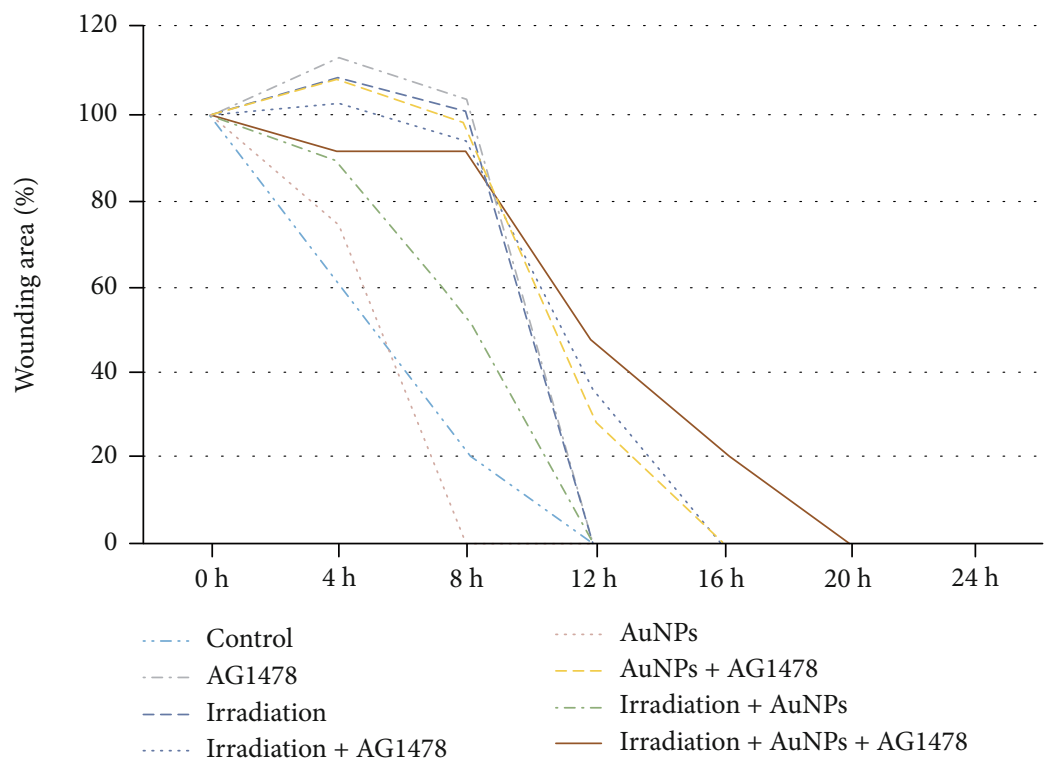

(b)

Figure 5: (a) Representative migration images. The scratched area was calculated from the area enclosed in green, which was automatically analyzed by Keyence's software BZ-H4M, according to the area of the central part of the scratch, excluding the missing part of the cells. (b) Adding AuNPs and/or irradiation to AG1478 reduced cell motility compared with AG1478 only. $Y$-axis values are expressed as percentages, with the control as $100 \%$. The data represent three independent experiments and are presented as means \pm standard deviation.

with EIs reduced the number of A431 cells compared with irradiation of gold nanoshells alone [39]. Dahai et al. reported that combining irradiation with either AgNP or $\mathrm{Ag} / \mathrm{C} 225$ reduced expression of DNA repair proteins [32]. These reports imply that EIs bound to AuNPs can impart high energy to DNA damage, which would be expected to increase cell damage and apoptosis. Another possible mechanism is radiation-induced reactive oxygen species (ROS) in the vicinity of the inhibitor-bound tumor cells.

A limitation of this study is that no factors other than cell viability and apoptosis (e.g., ROS production, oxidative stress, DNA damage, cell-cycle effects, and potential bystander effects) were evaluated. Further investigations into the effects of AuNPs conjugated to AG1478 on DNA repair, cell-cycle arrest, and autophagy in irradiation are needed. The current study showed that adding AuNPs enhanced the cytotoxicity of AG1478 and irradiation in vitro, but in vivo studies are required. AuNPs may remain in the body semipermanently, as no method for their metabolism and excretion has been established [40-42]; however, irradiation with AuNPs and EI may be a treatment option for HNSCC, especially oral cancers, as spatially focused treatment options, such as direct tumor injection and super selective arterial infusion therapy, are available.

\section{Conclusions}

AG1478 appeared to adsorb the surface of AuNPs by Raman spectroscopy. TEM images indicated the uptake of AuNPs into HSC-3 cells by AG1478 addition, but the AuNPs with and without AG1478 did not differ significantly. AuNPs can enhance the cytotoxic effects of irradiation and EGFR inhibitors against human HNSCC cells in vitro. This enhanced cytotoxicity was found to be caused by apoptosis with the AuNPs+irradiation combination and by inhibition of cell proliferation with the AuNPs +AG1478 combination.

\section{Data Availability}

The data used to support the findings of this study are available from the corresponding author upon request.

\section{Conflicts of Interest}

The authors declare that there is no conflict of interest regarding the publication of this paper. 


\section{Acknowledgments}

We thank Marla Brunker, from Edanz Group (https://enauthor-services.edanzgroup.com/ac), for editing a draft of this manuscript. The current work was supported by the Japan Society for the Promotion of Science, KAKENHI, Grant-in-Aid for Young Scientists (Grant No. 20K18693).

\section{References}

[1] J. Santini, J. L. Formento, M. Francoual et al., "Characterization, quantification, and potential clinical value of the epidermal growth factor receptor in head and neck squamous cell carcinomas," Head \& Neck, vol. 13, no. 2, pp. 132-139, 1991.

[2] J. R. Grandis, M. F. Melhem, W. E. Gooding et al., "Levels of TGF- $\alpha$ and EGFR Protein in Head and Neck Squamous Cell Carcinoma and Patient Survival," Journal of the National Cancer Institute, vol. 90, no. 11, pp. 824-832, 1998.

[3] M. T. Sheridan, T. O'Dwyer, C. B. Seymour, and C. E. Mothersill, "Potential indicators of radiosensitivity in squamous cell carcinoma of the head and neck," Radiation Oncology Investigations: Clinical and Basic Research, vol. 5, no. 4, pp. 180-186, 1997.

[4] K. K. Ang, B. A. Berkey, X. Tu et al., "Impact of epidermal growth factor receptor expression on survival and pattern of relapse in patients with advanced head and neck carcinoma," Cancer Research, vol. 62, no. 24, pp. 7350-7356, 2002.

[5] J. A. Bonner, P. M. Harari, J. Giralt et al., "Radiotherapy plus cetuximab for squamous-cell carcinoma of the head and neck," New England Journal of Medicine, vol. 354, no. 6, pp. 567-578, 2006.

[6] J. B. Vermorken, R. Mesia, F. Rivera et al., "Platinum-based chemotherapy plus cetuximab in head and neck cancer," New England Journal of Medicine, vol. 359, no. 11, pp. 11161127, 2008.

[7] H. K. Byeon, M. Ku, and J. Yang, "Beyond EGFR inhibition: multilateral combat strategies to stop the progression of head and neck cancer," Experimental \& Molecular Medicine, vol. 51, no. 1, pp. 1-14, 2019.

[8] E. S. Davidi, T. Dreifuss, M. Motiei et al., "Cisplatin-conjugated gold nanoparticles as a theranostic agent for head and neck cancer," Head \& Neck, vol. 40, no. 1, pp. 70-78, 2018.

[9] P. Joshi, S. Chakraborti, J. E. Ramirez-Vick et al., "The anticancer activity of chloroquine-gold nanoparticles against MCF-7 breast cancer cells," Colloids and Surfaces B: Biointerfaces, vol. 95, no. 1, pp. 195-200, 2012.

[10] H. Liu, M. Shen, J. Zhao et al., "Tunable synthesis and acetylation of dendrimer-entrapped or dendrimer-stabilized gold-silver alloy nanoparticles," Colloids and Surfaces. B, Biointerfaces, vol. 94, no. 1, pp. 58-67, 2012.

[11] A. F. de Faria, D. S. T. Martinez, S. M. M. Meira et al., "Antiadhesion and antibacterial activity of silver nanoparticles supported on graphene oxide sheets," Colloids and Surfaces B: Biointerfaces, vol. 113, no. 1, pp. 115-124, 2014.

[12] R. K. Mohanty, S. Thennarasu, and A. B. Mandal, "Resveratrol stabilized gold nanoparticles enable surface loading of doxorubicin and anticancer activity," Colloids and Surfaces B: Biointerfaces, vol. 114, no. 1, pp. 138-143, 2014.

[13] A. Kumar, X. Zhang, and X. J. Liang, "Gold nanoparticles: emerging paradigm for targeted drug delivery system," Biotechnology Advances, vol. 31, no. 5, pp. 593-606, 2013.
[14] A. Popovtzer, A. Mizrachi, M. Motiei et al., "Actively targeted gold nanoparticles as novel radiosensitizer agents: an in vivo head and neck cancer model," Nanoscale, vol. 8, no. 5, pp. 2678-2685, 2016.

[15] E. . E. Connor, J. Mwamuka, A. Gole, C. . J. Murphy, and M. . D. Wyatt, "Gold nanoparticles are taken up by human cells but do not cause acute cytotoxicity," Small, vol. 1, no. 3, pp. 325-327, 2005.

[16] C. Villiers, H. Freitas, R. Couderc, M. B. Villiers, and P. N. Marche, "Analysis of the toxicity of gold nano particles on the immune system: effect on dendritic cell functions," Journal of Nanoparticle Research, vol. 12, no. 1, pp. 55-60, 2010.

[17] O. Betzer, R. Meir, T. Dreifuss et al., "In-vitro optimization of nanoparticle-cell labeling protocols for in-vivo cell tracking applications," Scientific Reports, vol. 5, no. 1, article 15400, 2015.

[18] I. Elsayed, X. Huang, and M. Elsayed, "Selective laser photothermal therapy of epithelial carcinoma using anti-EGFR antibody conjugated gold nanoparticles," Cancer Letters, vol. 239, no. 1, pp. 129-135, 2006.

[19] C. I. Bargmann, M. C. Hung, and R. A. Weinberg, "The neu oncogene encodes an epidermal growth factor receptorrelated protein," Nature, vol. 319, no. 6050, pp. 226-230, 1986.

[20] L. Yunlong, S. Zhang, S. Dong et al., "Photothermolysis mediated by gold nanorods modified with EGFR monoclonal antibody induces Hep-2 cells apoptosis in vitro and in vivo," International Journal of Nanomedicine, vol. 17, no. 9, pp. 1931-1946, 2014.

[21] M. Zhang, H. S. Kim, T. Jin, and W. K. Moon, "Near-infrared photothermal therapy using EGFR-targeted gold nanoparticles increases autophagic cell death in breast cancer," Journal of Photochemistry and Photobiology B: Biology, vol. 170, no. 1, pp. 58-64, 2017.

[22] A. Otto, I. Mrozek, H. Grabhorn, and W. Akemann, "Surfaceenhanced Raman scattering," Journal of Physics: Condensed Matter, vol. 4, no. 5, pp. 1143-1212, 1992.

[23] M. Kerker, Selected Papers on Surface-Enhanced Raman Scattering, SPIE Optical Engineering Press, Bellingham, WA, 1990.

[24] S. Nie and S. R. Emory, "Probing single molecules and single nanoparticles by surface-enhanced Raman scattering," Science, vol. 275, no. 5303, pp. 1102-1106, 1997.

[25] A. Yamaguchi, Y. Utsumi, and T. Fukuoka, "Aggregation and dispersion of Au-nanoparticle-decorated polystyrene beads with SERS-activity using AC electric field and Brownian movement," Applied Surface Science, vol. 465, no. 1, pp. 405412, 2019.

[26] H. Segawa, T. Fukuoka, T. Itoh et al., "Rapid detection of hypnotics using surface-enhanced Raman scattering based on gold nanoparticle co-aggregation in a wet system," The Analyst, vol. 144, no. 6, pp. 2158-2165, 2019.

[27] H. Segawa, T. Fukuoka, T. Itoh et al., "Rapid detection of synthetic cannabinoids in herbal highs using surface-enhanced Raman scattering produced by gold nanoparticle coaggregation in a wet system," The Analyst, vol. 144, no. 23, pp. 6928-6935, 2019.

[28] T. Fukuoka, A. Yamaguchi, Y. Utsumi, R. Kuramoto, and Y. Mori, "Gold nanoparticles based nanosensors/nanobeacons fabricated by bottom-up method for surface enhanced raman scattering," Bunseki-Kagaku, vol. 66, no. 12, pp. 919-923, 2017.

[29] T. Itoh and Y. S. Yamamoto, "Recent topics on single-molecule fluctuation analysis using blinking in surface-enhanced 
resonance Raman scattering: clarification by the electromagnetic mechanism," The Analyst, vol. 141, no. 17, pp. 50005009, 2016.

[30] S. Teraoka, Y. Kakei, M. Akashi et al., "Gold nanoparticles enhance X-ray irradiation-induced apoptosis in head and neck squamous cell carcinoma in vitro," Biomedical Reports, vol. 9, no. 5, pp. 415-420, 2018.

[31] L. Monteiro, S. Ricardo, M. Delgado, F. Garcez, B. do Amaral, and C. Lopes, "Phosphorylated EGFR at tyrosine 1173 correlates with poor prognosis in oral squamous cell carcinomas," Oral Diseases, vol. 20, no. 2, pp. 178-185, 2014.

[32] D. Yu, Y. Zhang, H. Lu, and D. Zhao, "Silver nanoparticles coupled to anti-EGFR antibodies sensitize nasopharyngeal carcinoma cells to irradiation," Molecular Medicine Reports, vol. 16, no. 6, pp. 9005-9010, 2017.

[33] A. T. N. Lam, J. Yoon, E.-O. Ganbold et al., "Colloidal gold nanoparticle conjugates of gefitinib," Colloids and Surfaces B: Biointerfaces, vol. 123, no. 1, pp. 61-67, 2014.

[34] A. T. N. Lam, J. Yoon, E.-O. Ganbold et al., "Adsorption and desorption of tyrosine kinase inhibitor erlotinib on gold nanoparticles," Journal of Colloid and Interface Science, vol. 425, no. 1, pp. 96-101, 2014.

[35] Y. Qian, M. Qiu, Q. Wu et al., "Enhanced cytotoxic activity of cetuximab in EGFR-positive lung cancer by conjugating with gold nanoparticles," Scientific Reports, vol. 4, no. 1, article 7490, 2014.

[36] H. J. Jeon, B. B. R. Choi, K. H. Park, D. S. Hwang, U. K. Kim, and G. C. Kim, "Induction of melanoma cell-selective apoptosis using anti-HER2 antibody-conjugated gold nanoparticles," Yonsei Medical Journal, vol. 60, no. 6, pp. 509-516, 2019.

[37] T. Kubota, S. Kuroda, N. Kanaya et al., "HER2-targeted gold nanoparticles potentially overcome resistance to trastuzumab in gastric cancer," Nanomedicine, vol. 14, no. 6, pp. 19191929, 2018.

[38] W. Jiang, B. Y. S. Kim, J. T. Rutka, and W. C. W. Chan, "Nanoparticle-mediated cellular response is size-dependent," Nature Nanotechnology, vol. 3, no. 3, pp. 145-150, 2008.

[39] M. P. Melancon, W. Lu, Z. Yang et al., "In vitro and in vivo targeting of hollow gold nanoshells directed at epidermal growth factor receptor for photothermal ablation therapy," Molecular Cancer Therapeutics, vol. 7, no. 6, pp. 1730-1739, 2008.

[40] G. S. Terentyuk, E. Panfilova, V. Khanadeev et al., "Gold nanorods with a hematoporphyrin-loaded silica shell for dualmodality photodynamic and photothermal treatment of tumors in vivo," Nano Research, vol. 7, no. 3, pp. 325-337, 2014.

[41] F. Yang, C. Jin, D. Yang et al., "Magnetic functionalised carbon nanotubes as drug vehicles for cancer lymph node metastasis treatment," European Journal of Cancer, vol. 47, no. 12, pp. 1873-1882, 2011.

[42] X. Huang and M. A. El-Sayed, "Gold nanoparticles: Optical properties and implementations in cancer diagnosis and photothermal therapy," Journal of Advanced Research, vol. 1, no. 1, pp. 13-28, 2010. 